\title{
LOCAL PERFORMANCE OPTIMIZATION FOR A CLASS OF REDUNDANT EIGHT-DEGREE-OF-FREEDOM MANIPULATORS
}

\author{
Dr. Robert L. Williams II \\ NASA Langley Research Center \\ Hampton, VA 23681-0001
}

\begin{abstract}
Local performance optimization for joint limit avoidance and manipulability maximization is presented using the Jacobian matrix pseudoinverse and objective function gradient projection into the Jacobian null space. Real-time performance optimization is achieved for a member of the class of 8-joint redundant manipulators having a 3-axis spherical shoulder, a single elbow joint, and a 4-axis spherical wrist. Symbolic solutions are used for both full and partitioned Jacobian matrix pseudoinverses and objective function gradients. Results are presented to demonstrate the effectiveness of the performance optimization. A kinematic limitation of this class of manipulators and its effect on redundancy resolution is discussed.

The partitioned solution is desirable because of low computation. The partitioned solution is suboptimal compared to the full solution because translational and rotational terms are optimized separately, but the difference is not significant. Singularity analysis reveals the full and partitioned solutions share the same physical manipulator singularities. The partitioned solution is illconditioned in smaller neighborhoods of the singularities than the full solution.
\end{abstract}

\section{INTRODUCTION}

Kinematically redundant manipulators, those with more degrees-of-freedom than task constraints, can be used in a secondary task of performance optimization in addition to the primary task of providing a Cartesian trajectory. Whitney [13] derived the pseudoinverse solution for the primary task in the framework of the resolved motion rate algorithm. Liegeois [9] suggested local redundancy resolution using the Moore-Penrose pseudoinverse [12] to solve the primary task and projecting the gradient of an objective function into the null space of the Jacobian matrix for the secondary task. A good review of pseudoinverse-based local redundancy resolution is given in [10] and [7].

Many authors who found that Liegeois' method is too slow for real-time application to 3-D redundant manipulators developed alternative methods focusing on reduced computational requirement (e.g. [4], [1], [8]). The approach in the current paper is to apply Liegeois' method using symbolic pseudoinverses for both full and partitioned Jacobian matrices.

Other authors have investigated the use of partitioned or symbolic methods in redundancy resolution. Kircanski and Petrovic [6] decompose a manipulator into redundant and non-redundant subassemblies; the redundant joints are solved by singular value decomposition or equivalent methods, while the nonredundant joints are solved analytically. Holt [5] presents numerical computation of the "approximate pseudoinverse" for a PUMA near singularities using the wrist-partitioned Jacobian matrix. Chevallereau and Khalil [2] present symbolic pseudoinverse calculations for non-redundant manipulators at singularities and for a 6-DOF manipulator on a linear track. Podhorodeski, et. al. [11] use screw theory in an orthogonal decomposition of a redundant manipulator Jacobian matrix to determine analytical expressions for the particular solution and the null space basis.

The current paper describes real-time local performance optimization for a class of 8-revolute joint redundant manipulators having a 3-axis spherical shoulder $S$, a single elbow joint $E$, and a 4-axis spherical wrist $W$, as pictured in Fig. 1. The Advanced Research Manipulator II (ARMII) is a specific 8-axis manipulator in this class for which the theory of this paper has been applied. The objective functions for the secondary optimization task are joint limit avoidance, manipulability maximization (thus singularity avoidance), and a combination of the two. Symbolic pseudoinverses and objective function gradients are used to solve the problem for full and partitioned Jacobian matrices. Singularity analysis is presented to determine if the partitioned solution has algorithmic singularities. The accuracy of the partitioned and the full solutions is compared in the neighborhood of the singularities. Finally, a kinematic design limitation of this class of 8joint arms is discussed, along with its effect on local redundancy optimization. 


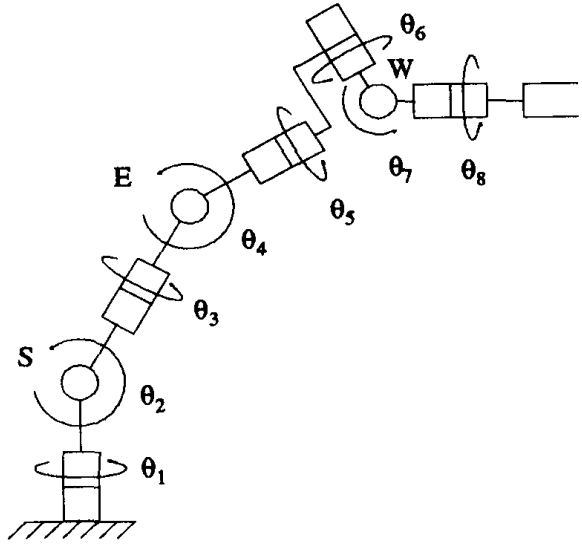

Figure 1

Specific Class of 8-Axis Manipulators

\section{LOCAL REDUNDANCY OPTIMIZATION}

\subsection{General Redundant Solution}

The resolved motion rate algorithm [13] is based on the following equation, where joint rates $\dot{\theta}$ are mapped to Cartesian velocities $\dot{X}$ by the Jacobian matrix $J$.

$$
\dot{X}=\boldsymbol{J} \dot{\theta}
$$

In Eq. $1, \dot{X} \in \Re^{m \times 1}, J \in \Re^{m \times n}$, and $\dot{\theta} \in \Re^{n \times 1}$, where $m$ is the dimension of the task space ( $m=6$ for spatial Cartesian control) and $n$ is dimension of the joint space ( $n=8$ in this paper). To command a trajectory $\dot{X}$ to a manipulator, $\dot{\theta}$ must be solved.

A kinematically redundant manipulator has more degrees of freedom than the required task space degrees of freedom, i.e. $m<n$. In this case, Eq. 1 is underconstrained, and infinite solutions for $\dot{\theta}$ generally exist. A general resolved motion rate solution is expressed by the following [9].

$\dot{\theta}=J^{*} \dot{X}+k\left(I_{n}-J^{*} J\right) \nabla H$

The first term of Eq. 2 is the particular solution. The matrix $J^{*}$ is the Moore-Penrose pseudoinverse [12] of the Jacobian matrix, which provides the least-squares solution of Eq. 1 to achieve the Cartesian velocity command, called the primary task. The second term is the homogeneous solution, which causes zero motion of the end-effector (self-motion). The linear operator $\left(I_{n}-J^{*} J\right)$ projects an arbitrary vector onto the null space of the Jacobian matrix. To optimize performance criteria (secondary task), the gradient of a objective function of joint angles $\nabla H$ is used [9]. The gain $k$ is positive to maximize $H$ and negative to minimize $H$.

\subsection{Eight-Axis Redundant Solution}

This section presents adaptation of Eq. 2 to the eightjoint manipulators pictured in Fig. 1. This manipulator type may be viewed as two subassemblies: an arm portion $\theta_{1}, \theta_{2}, \theta_{3}, \theta_{4}$ and a spherical wrist $\theta_{5}, \theta_{6}, \theta_{7}, \theta_{8}$ centered at $W$. The arm subassembly consists of a 3 -jointed spherical shoulder centered at $S$, and a single elbow joint at $E$. The 4-jointed wrist mechanism is of roll - yaw pitch - roll configuration. Equations in this section are given with minimum development; for details, see [16]. Two solutions are presented: full and partitioned. For each solution, the Jacobian matrix referred to the elbow joint frame is used because it is least complex [14].

The ARMII is a specific 8-axis manipulator of the class in Fig. 1. The theory of this paper has been implemented in real-time ( $33 \mathrm{~Hz}$ update) on the ARMII. For more information on the ARMII, see [14] and [16].

2.2.1. Reduced Jacobian Solution. For manipulators with a spherical wrist, spherical shoulder, and single elbow joint, the elbow joint rate is solved independently of the remaining unknowns because the length of reach from $S$ to $W$ is a function of only the elbow joint angle. The full 6 by 8 Jacobian solution is reduced to 5 by 7 by removing column 4 and row 1 . Equations 3 and 4 represent the general full Jacobian solution for the manipulator of Fig. 1.

$$
\begin{aligned}
& \dot{\theta}_{4}=\frac{-1}{d_{5}}\left[\dot{x}+\frac{\left(d_{3} c_{4}+d_{5}\right)}{d_{3} s_{4}} \dot{y}\right] \\
& \dot{\theta}_{i \neq 4}=J_{5 \times 7}^{*}\left(\dot{X}_{1}-J_{4} \dot{\theta}_{4}\right)+k\left(I_{7}-J_{5 \times 7}^{*} J_{5 \times 7}\right) \nabla H_{i \neq 4}
\end{aligned}
$$

In Eq. 3, $d_{3}$ and $d_{5}$ are lengths $S E$ and $E W, \dot{x}$ and $\dot{y}$ are wrist Cartesian velocity commands expressed in the elbow frame, and $s_{4}$ and $c_{4}$ are the sine and cosine of the elbow joint angle $\theta_{4}$. The vector $\dot{X}_{1}$ is the Cartesian velocity command and $J_{4}$ is the fourth column of the Jacobian matrix, each with row 1 removed. The pseudoinverse of the reduced Jacobian matrix, $J_{5 \times 7}^{*}$, was derived with a symbolic manipulation program using $J^{*}=J^{T}\left(J J^{T}\right)^{-1}$ [15]. No loss of generality is incurred by using Eq. 3. If the geometric relationship is not exploited, the pseudoinverse of the 6 by 8 Jacobian matrix always yields the Eq. 3 value for $\dot{\theta}_{4}$

The null space projection operator in the second term of Eq. 4 is a square matrix of order 7, using the reduced 
Jacobian matrix. The homogeneous term for $\dot{\theta}_{4}$ is zero, due to the geometric elbow constraint: any addition to the particular solution, Eq. 3, would cause a deviation in the commanded trajectory. The fourth term of the constraint function gradient is excluded, as explained below.

No loss of generality is incurred by using the second term of Eq. 4 for the homogeneous solution to Eq. 1. In general, $J J^{*}=I_{m}$, but $J^{*} J \neq I_{n}$. However, due to the geometric elbow constraint of the 8-axis arm, the fourth row and column of $J^{*} J$ are the same as the fourth row and column of the Identity matrix, when $J$ is the full $6 \mathrm{x}$ 8 Jacobian matrix. The null space projection matrix thus has zeros for the fourth row and column in this case. This has two effects: 1) Because the fourth row is zero, a homogeneous term for $\dot{\theta}_{4}$ does not exist; 2) Because the fourth column is zero, the partial derivative of a constraint function with respect to $\theta_{4}$ never adds to the homogeneous terms for the other joint rates.

2.2.2. Partitioned Reduced Jacobian Solution. A partitioned solution of $\mathrm{Eq}$. 1 was developed to reduce computation. The full symbolic particular solution requires 25 times more computation than the partitioned solution [15]. For a manipulator with a spherical wrist, Eq. 1 is written in the following partitioned form.

$$
\left\{\begin{array}{l}
v \\
\omega
\end{array}\right\}=\left[\begin{array}{cc}
J_{U L} & 0 \\
J_{L L} & J_{L R}
\end{array}\right]\left\{\begin{array}{l}
\dot{\theta}_{A} \\
\dot{\theta}_{W}
\end{array}\right\}
$$

The vectors $v$ and $\omega$ are the translational and rotational Cartesian velocity commands. The Jacobian matrix is partitioned into 3 by 4 submatrices. The vector $\dot{\theta}_{A}$ represents the arm joint rates 1 through 4 , and $\dot{\theta}_{W}$ the wrist joint rates 5 through 8 . First $\dot{\theta}_{4}$ is determined from Eq. 3. Again, the homogeneous solution for $\dot{\theta}_{4}$ is zero. The total solution for the remaining arm joint rates is:

$\dot{\theta}_{A, i \neq 4}=J_{1 U L 4}^{*}\left(v_{1}-J_{U L 4} \dot{\theta}_{4}\right)+k_{A}\left(I_{3}-J_{1 U L 4}^{*} J_{1 U L 4}\right) \nabla H_{A, i \neq 4}$

$J_{1 U L 4}^{*}$ is the 3 by 2 pseudoinverse of $J_{U L}$ with column 4 and row 1 removed, and $v_{1}$ is $v$ with row 1 removed. $J_{U L 4}$ is column 4 of $J_{U L}$ with row 1 removed. For the same reasons stated for the full solution above, no loss of generality is incurred by using Eq. 3 and reducing $J_{U L}$ to $J_{1 U L 4}$ for both particular and homogeneous translational solutions.

The total wrist joint rate solution is Eq. 7. Symbolic $J_{1 U L 4}^{*}$ and $J_{L R}^{*}$ are given in [16]. In Eq. 7, the vector $\dot{\theta}_{A T}$ is the total arm joint rate solution, Eqs. 3 and 6 .
$\dot{\theta}_{W}=J_{L R}^{*}\left(\omega-J_{L L} \dot{\theta}_{A T}\right)+k_{W}\left(I_{4}-J_{L R}^{*} J_{L R}\right) \nabla H_{W}$

2.2.3 Eight-Axis Singularity Analysis. This section summarizes singularity conditions for the manipulator of Fig. 1. For details and physical interpretation, see [16]. For the partitioned case, singularity analysis is presented below. The calculation of $\dot{\theta}_{4}$ in Eq. 3 fails when $d_{5}=0$ (which is not possible) or when $\theta_{4}=0, \pi$. The condition for the remaining translational joints is Eq. 8 .

$\left|J_{1 U L 4} J_{1 U L 4}^{T}\right|=d_{3}{ }^{2} s_{4}{ }^{2} D_{U L}=0$

$D_{v L}=\left(d_{3}^{2}+d_{5}^{2}\right) s_{2}^{2}+2 d_{5}\left(s_{2} c_{4}+c_{2} c_{3} s_{4}\right)\left(d_{3} s_{2}+d_{5} c_{2} c_{3} s_{4}\right)$

The wrist joints are singular when Eq. 9 is satisfied.

$\left|J_{L R} J_{L R}^{T}\right|=2\left(1-s_{6}{ }^{2} s_{7}^{2}\right)=0$

Table I summarizes the four singularity conditions. The zero reference for all joint angles is when the manipulator in Fig. 1 is vertical.

Table I: Singularity Conditions

\begin{tabular}{|l|l|}
\hline 1 & $\theta_{4}=0,180^{\circ}$ \\
\hline 2 & $\theta_{2}=0,180^{\circ}$ and $\theta_{3}= \pm 90^{\circ}$ \\
\hline 3 & $\theta_{2}=\theta_{4}=0,180^{\circ}$ \\
\hline 4 & $\theta_{6}= \pm 90^{\circ}$ and $\theta_{7}= \pm 90^{\circ}$ \\
\hline
\end{tabular}

Entries 1 and 3 are workspace limit singularities, while entries 2 and 4 are workspace interior singularities. The above conditions were derived from the partitioned solution. The symbolic $\left|J_{5 \times 7} J_{5 \times 7}^{T}\right|$ was too complex for analysis. However, numerical computer searches indicated that the full Jacobian shares all four singularity conditions from Table I. Furthermore, no additional singularities exist for the full case. This behavior is expected because of the spherical wrist.

In the neighborhood of manipulator singularities, both full and partitioned symbolic solutions become illconditioned. Numerical singular value decomposition solutions can be used in these regions, but general trajectories are not possible due to the singular condition.

\section{2,3 Objective Functions}

This section presents objective functions to optimize performance criteria of a redundant manipulator via the homogeneous solution. Two criteria have been implemented for the full and partitioned solutions: joint limit avoidance and manipulability maximization 
(singularity avoidance). The gradients of the objective functions have been derived symbolically.

The following function was proposed by Liegeois [9] to avoid joint limits:

$$
H_{J}(\theta)=\sum_{i=1}^{n}\left(\frac{\theta_{i}-\theta_{c i}}{\Delta \theta_{i}}\right)^{2}
$$

where $\theta_{i}$ is the current value for joint $i, \theta_{c i}$ is the center of travel for joint $i$, and $\Delta \theta_{i}$ is half the range of travel for joint $i$. This function is minimized for joint limit avoidance. Equation 10 is defined for the full case; for the partitioned case, $H_{A}$ is defined for $i=1$ to 4 , and $H_{w}$ for $i=5$ to 8 . [17]

Yoshikawa's definition of manipulability is as follows

$$
H_{M}(\theta)=\sqrt{\left|J J^{T}\right|}
$$

This function must be maximized to avoid singularities and ensure that the manipulator operates away from singular configurations in general. For the full homogeneous solution, the function is $H_{M}=\sqrt{\left|J_{5 \times 7} J_{5 \times 7}^{T}\right|}$ and for the partitioned case $H_{A}=\sqrt{\left|J_{1 U L 4} J_{1 U L 4}^{T}\right|}$ and $H_{W}=\sqrt{\left|J_{L R} J_{L R}^{T}\right|}$.

\section{RESULTS}

\subsection{Local Optimization Results}

This section presents local redundancy optimization results for the full solution. Results are given for joint limit avoidance, manipulability maximization, and a combination of the two. Units are MKS. The joint limit constraint function $H_{J}$ is dimensionless, and units for manipulability $H_{M}$ are $m^{2}$.

3.1.1 Joint Limit Avoidance (JLA). The constraint function is $\mathrm{Eq}$. 10, with $n=8$. The $i=4$ term is included, but elbow joint limits cannot be avoided because the homogeneous term for the elbow joint is zero; see Section 2.2.1. The trajectory is an end-effector roll, $\dot{X}=\{0,0,0,0,0,0.4\}^{T}$; the starting configuration is $\theta=\{0,-30,0,-70,0,0,-50,0\}^{T}$. Figure 2 compares $H_{J}$ for the particular solution only ( $k=0$ in Eq. 4) and with joint limit avoidance $(k=-0.5)$. For this trajectory, $\theta_{5}$ hits a limit at 9.5 seconds with $k=0$, but avoids the limit when $k=-0.5$. The Cartesian error for $k=0$ is 200 $\mathrm{mm}$ after 20 seconds due to the joint limit.

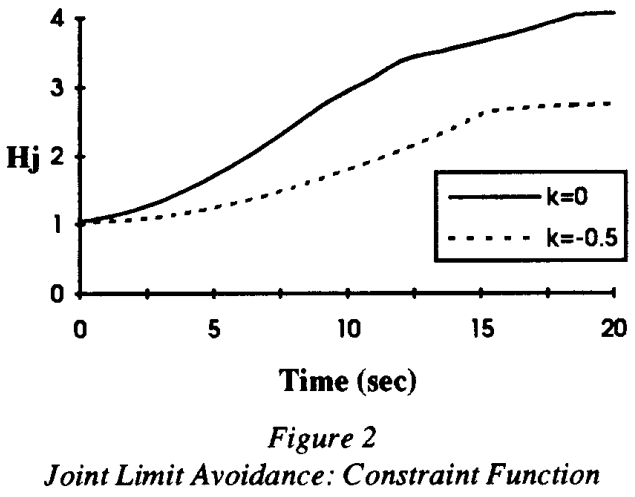

3.1.2 Manioulability Maximization (MM). The constraint function is $H_{M}$, defined following Eq. 11 . The Cartesian trajectory is $\dot{X}=\{0,-0.01,0,0,0,0\}^{T}$; the initial manipulator configuration is near both arm and wrist singularities, $\theta=\{0,-10,75,-70,0,-80,-90,0\}^{T}$. Figure 3 shows $H_{M}$ for the particular solution only $(k=0)$ and with manipulability maximization $(k=1)$. Without manipulability maximization, the trajectory drives the manipulator further into the wrist singularity, and the manipulability is near zero for the entire move. The optimized case avoids the singularity, increasing the manipulability rapidly and maintaining it at a high level while achieving the trajectory.

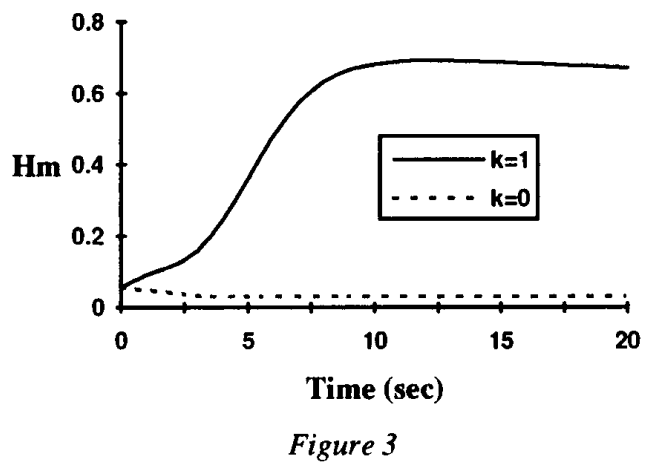

Manipulability Maximization: Constraint Function

3.1.3 Combined Optimization. Experiments with manipulability maximization using the full solution revealed that regions exist where the local maximum for $H_{M}$ lies outside of joint limits. Such cases indicate that it is sometimes necessary to combine optimization criteria. The objective function maximizes manipulability and avoids joint limits:

$$
H(\theta)=k_{M} H_{M}+k_{J} H_{J}
$$


The trajectory is $\dot{X}=\{0.01,0.01,0.01,0,0,0\}^{T}$; $\theta=\{0,-10,85,-70,0,-80,-90,0\}^{T}$ is the initial manipulator configuration. With manipulability maximization only (MM, $k_{M}=1, k_{J}=0$ ), a joint limit for $\theta_{7}$ is reached at 9 seconds, which causes the Cartesian error to increase rapidly (Fig. 4). Using the combined optimization of Eq. 13, the manipulability is not as high, but no joint limits are encountered (MM+JLA, $k_{M}=1, k_{J}=-1$ ).

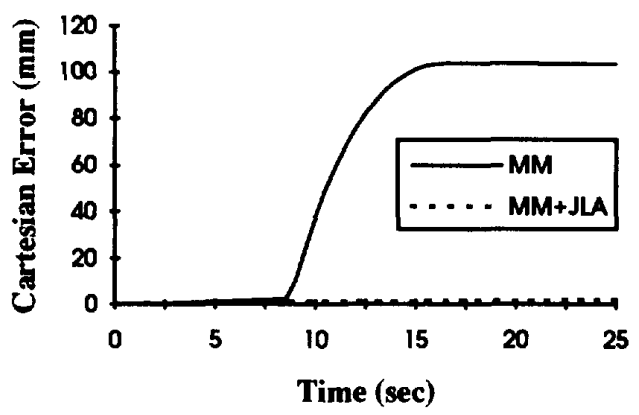

Figure 4

Combined Optimization: Cartesian Error

\subsection{Comparison of Partitional and Full Solutions}

The results presented above are for the full solution. The partitioned solution is suboptimal because the particular and homogeneous solutions are optimized separately over translational and rotational parts. The difference is not significant as results in [16] show. This section summarizes the comparison in four areas. 1) Particular solutions: the full solution produces the leastsquares solution for joint rates, but the partitioned solution is only slightly higher. One case shows a $2.5 \%$ maximum difference, which decreases in singular regions. 2) Joint limit avoidance: The partitioned solution applied to the trajectory of Section 3.1.1 yields an $H_{J}$ nearly identical to the full case. 3) Manipulability maximization: The wrist manipulability $H_{W}$ tends to be significantly higher, while the arm manipulability $H_{A}$ is somewhat lower than the full manipulability $H_{M}$. 4) Accuracy of the partitioned solution: The full solution is ill-conditioned in a greater neighborhood of the internal singularities (lines 2 and 4 in Table I) than the arm and wrist partitioned solutions.

\section{EIGHT-AXIS ARM DESIGN LIMITATION}

This section discusses a limitation in the eight-axis arm design regarding redundancy optimization. As discussed in Section 2.2.1, the length of reach from shoulder to wrist for manipulators with a spherical wrist, spherical shoulder, and a single eltow joint is a function of only the elbow joint angle. The limitation in this design is that the elbow joint can only be used to satisfy the primary task, the Cartesian trajectory. The elbow joint cannot be used in the secondary task of manipulator performance optimization because it does not influence the self-motion of the arm. Although the arm has two redundant degrees-of-freedom, it has only one mode of self-motion, the elbow-orbit about the line $\overline{S W}$. This self-motion is achieved by seven-DOF manipulators with only one redundant freedom.

In order to provide two modes of self-motion with two redundant joints, and to allow the elbow joint to influence performance optimization, kinematic design modifications to the arm of Fig. 1 are discussed. One altemative is to reconfigure joint 5 so it is parallel to joint 4. This design has two modes of self-motion, the original elbow-orbit, plus a four-bar motion in the plane of the links connecting $S$ and $W$.

Another design alternative is to reconfigure joint 5 as a prismatic joint acting along the line connecting joint 4 and $W$ in Fig. 1 . In this case, the subassembly connecting $S$ to $W$ provides a slider-crank mode of selfmotion.

A drawback of these proposed design alternatives is that the four-joint spherical wrist subassembly of the original arm is reduced to three joints. The partitioned solution applied to the new designs would not be useful because the wrist subassembly is no longer redundant, so no wrist performance optimization using self-motion is possible. A nine-jointed altemative has a second elbow revolute joint (parallel to joint 4) between joints 4 and 5 of Fig. 1. (Alternatively, the prismatic joint can be used).

There are tradeoffs among the original design of Fig. 1 and the three proposed redesigns. The Fig. 1 design has simpler kinematics, joint 4 variables solved independently, and allows an efficient partitioned solution. However, there is no null space for joint 4 , and only one mode of self-motion. The 8-joint redesign concepts provide two modes of self-motion, a null space term associated with $\dot{\theta}_{4}$, but no four-jointed spherical wrist to allow a general partitioned solution with reduced computation. The 9-joint redesign provides all desired attributes, at the cost of an extra joint.

\section{CONCLUSION}

This paper presents local redundancy optimization for a class of eight-axis redundant arms. The theory has been implemented on the Advanced Research Manipulator II (ARMII). The performance constraints for the secondary task optimization are joint limit 
avoidance, manipulability maximization and a combination of the two. Results are presented to show the effectiveness of the redundancy optimization.

The methods used in this paper are well known from the redundant manipulator literature. The contributions of this paper are four-fold. 1) Real-time local performance optimization for an experimental eight-axis manipulator is demonstrated. 2) A kinematic design limitation of this class of eight-axis arms is explained. The elbow angle participates only in the primary task, and cannot affect the secondary optimization task. There are two redundant degrees of freedom but only one mode of self-motion. The geometry is suited for low computation redundancy resolution, but the tradeoff is reduced versatility. 3) Symbolic pseudoinverses and objective function gradients are used for both full and partitioned solutions. 4) A partitioned solution is applied to reduce computational requirements. The partitioned solution is suboptimal because translational and rotational terms are optimized separately for both primary and secondary tasks, but results presented in [16] show the difference is not significant. Singularity analysis reveals that all partitioned singularities also exist for the full solution and thus no algorithmic singularities exist for the partitioned solution. The partitioned solution is ill-conditioned in smaller neighborhoods of singularities than the full solution.

\section{REFERENCES}

[1] Baillieul, J., "Kinematic Programming Alternatives for Redundant Manipulators", 1985 IEEE Intemational Conference on Robotics and Automation, pp. 722-728.

[2] Chevallereau, C., and Khalil, W., "Efficient Method for the Calculation of the Pseudo Inverse Kinematic Problem", 1987 IEEE International Conference on Robotics and Automation, Vol. 3, pp. 1842-1848.

[3] Craig, J.J., Introduction to Robotics: Mechanics and Control, Addison-Wesley Publishing Co., Reading, MA, 1988.

[4] Dubey, R.V., Euler, J.A., and Babcock, S.M., "RealTime Implementation of an Optimization Scheme for Seven-Degree-of-Freedom Redundant Manipulators", IEEE Transactions on Robotics and Automation, Vol. 7, No. 5, October 1991, pp. 579-588.

[5] Holt, K., "Inertial-Space Disturbance Rejection for Robotic Manipulators", Master of Engineering Thesis, Rensselaer Polytechnic Institute, November, 1992.

[6] Kircanski, M.V., and Petrovic, T.M., "Inverse Kinematic Solution for a 7 dof Robot with Minimal Computational Complexity and Singularity Avoidance", 1991 IEEE Intemational Conference on Robotics and Automation, pp. 2664-2669.
[7] Klein, C.A., and Huang, C.H., "Review of Pseudoinverse Control for use with Kinematically Redundant Manipulators", IEEE Transactions on Systems, Man, and Cybernetics, Vol. SMC-13, No. 3, March-April 1983, pp. 245-250.

[8] Lee, S., and Bejczy, A.K., "Redundant Arm Kinematic Control Based on Parameterization", 1991 IEEE International Conference on Robotics and Automation, pp. 458-465.

[9] Liegeois, A., "Automatic Supervisory Control of the Configuration and Behavior of Multibody Mechanisms", IEEE Transactions on Systems, Man, and Cybemetics, Vol. SMC-17, No. 12, December 1977, pp. 868-871.

[10] Nenchev, D.N., "Redundancy Resolution through Local Optimization: A Review", Journal of Robotic Systems, Vol. 6, No. 6, 1989, pp. 769-798.

[11] Podhorodeski, R.P., Goldenberg, A.A., and Fenton, R.G., "Resolving Redundant Manipulator Joint Rates and Identifying Special Arm Configurations using Jacobian Null-Space Bases", IEEE Transactions on Robotics and Automation, Vol. 7, No. 5, October 1991, pp. 607-618.

[12] Strang, G., Linear Algebra and its Applications, Harcourt Brace Jovanovich, Publishers, San Diego, CA, 1988.

[13] Whitney, D.E., "Resolved Motion Rate Control of Manipulators and Human Prostheses", IEEE

Transactions on Man-Machine Systems, Vol. 10, 1969, pp. 47-53.

[14] Williams, R.L., "Kinematic Equations for the Eight Degree-of-Freedom Advanced Research Manipulator II (ARMII)", NASA TM 4377, NASA Langley Research Center, June, 1992.

[15] Williams, R.L., and Aldridge, H.A., "Comparison of Pseudoinverse Computation Methods to Control an Eight-Degree-of-Freedom Manipulator", Submitted to the Third National Conference on Applied Mechanisms and Robotics, Cincinnati, OH, November, 1993.

[16] Williams, R.L., "Local Redundancy Optimization for a Class of Eight Degree-of-Freedom Manipulators Using Symbolic Pseudoinverses", Pending NASA TP L17289, NASA Langley Research Center, 1993.

[17] Yoshikawa, T., "Analysis and Control of Robot Manipulators with Redundancy", Robotics Research, eds. M. Brady and R. Paul, MIT Press, 1984, pp. 735747. 